with no antiepileptic drug therapy, in a study at KSU Medical School, Turkey. Nitrite and nitrate levels, metabolites of nitric oxide, were significantly higher in both valproic acid and carbamazepine-treated groups compared to controls $(\mathrm{p}<0.01)$, and nitrate but not nitrite was significantly higher in the valproic acid-treated group compared to the carbamazepine group $(\mathrm{p}<0.01)$. Serum drug levels did not correlate with nitrite and nitrate levels. The authors suggest that valproic acid and carbamazepine might exert an antiepileptic effect through nitric oxide. (Karabiber H, Yakinci C, Durmaz Y et al. Serum nitrite and nitrate levels in epileptic children using valproic acid or carbamazepine. Brain Dev January 2004;26:15-18). (Respond: Dr Hamza Karabiber, KSU Medical School, Department of Pediatrics, 46050 Kahramanmaras, Turkey).

COMMENT. Nitrite and nitrate levels are used as indicators of nitric oxide (NO) levels in serum. NO has been found to have an anticonvulsant effect in animal studies. The above report suggests that the anticonvulsants, valproic acid and carbamazepine, may have antiepileptic effects through $\mathrm{NO}$ accumulation.

Valproic acid (VPA) blood gene expression patterns and epilepsy. The expression of 461 genes was altered in VPA-treated compared with drug-free children with epilepsy, in a study at the University of Cincinnati (Tang Y et al. Acta Neurologica Scandinavica March 2004;109:159-168). A new mechanism of action of VPA is suggested that involves downregulation of serine threonine kinases and a separate genomic profile that correlates with VPA-induced seizure freedom.

\title{
MEMORY PERFORMANCE WITH INTRACTABLE EPILEPSY
}

The cognitive and psychological predictors of everyday memory were investigated in 37 children (mean age 13.7 years, range 7.3-17.9 years) with medically intractable epilepsy treated at the Hospital for Sick Children, Toronto, Canada. Only a parent-report measure of attention (Child Behavior Checklist Attention) significantly predicted everyday memory ratings. Standard tests of attention, intelligence, visual and verbal memory, working memory, and mood/emotional state were not predictive of everyday memory. (Kadis DS, Stollstorff M, Elliott I et al. Cognitive and psychological predictors of everyday memory in children with intractable epilepsy. Epilepsy Behav 2004;5:37-43). (Respond: Dr Darren S Kadis, Hospital for Sick Children, Toronto, Ontario, Canada).

COMMENT. Everyday memory deficits in children with epilepsy are secondary to attentional problems. They differ from that of adults whose everyday memory correlates with standard psychological measures. Memory deficits in adults with epilepsy have also been correlated with anxiety and/or depression, age and duration of the seizure disorder.

Memory impairment with febrile seizures. Adult rats that had been subjected to repetitive brief, experimental febrile seizures (FS) at days 10 to 12 postpartum, showed long-term memory deficits as assessed by the Morris water maze test (Chang Y-C et al. Ann Neurol Dec 2003;54:706-718). These results raise concerns about long-term cognitive consequences of even brief repetitive FS during infancy. Despite limitations, immature animal experimental FS can provide useful correlations with the clinical FS, its mechanism and 
functional sequelae (Baram TZ. Editorial, Ann Neurol Dec 2003;54:701-704; Millichap JG. Studies in febrile seizures I. Height of body tempeature as a measure of the febrile seizure threshold. Pediatrics Jan 1959;23:76-85).

Effects of seizure-related activity on cognitive function. Paroxysmal epileptic activity, the acute effects of minor seizures and epileptic EEG discharges, caused deficits in cognitive processes, alertness and mental speed, in children with short nonconvulsive seizures, and over time, affected educational achievement (Aldenkamp A, Arends J. Epilepsia January 2004;45:54-63).

\section{RISK FACTORS FOR RECURRENCE AFTER FIRST SEIZURE}

Recurrence after a first unprovoked cryptogenic/idiopathic seizure was studied in 213 children followed at FCM-Unicamp, Campinas, SP, Brazil. Recurrence occurred in 34\% of patients at a mean interval of 12 months. An abnormal EEG was a significant risk factor for seizure recurrence. Small calcifications found in $9.5 \%$ of CTs performed in 182 patients were not a predictor for recurrence. (Scotoni AE, Manreza MLG, Guerreiro MM. Recurrence after a first unprovoked cryptogenic/idiopathic seizure in children: a prospective study from Sao Paulo, Brazil. Epilepsia February 2004;45:166-170). (Reprints: Dr MM Guerreiro, Department of Neurology, FCM-Unicamp, PO Box 6111, 13083-970 Campinas, SP, Brazil).

COMMENT. EEG but not $\mathrm{CT}$ abnormalities are predictors of seizure recurrence after a first unprovoked cryptogenic/idiopathic seizure.

In 82 patients with childhood-onset cryptogenic localization-related epilepsies controlled for 3 years or more, seizures recurred in $8(9.8 \%)$ after withdrawal of antiepileptic drugs. (Ohta H et al. Brain Dev 2004;26:19-25). Factors correlating with higher rates of seizure relapse included: 6 years of age or higher at epilepsy onset; 15 years of age or higher at start of AED withdrawal; 5 years or more from start of AED treatment to seizure control; 5 or more seizures before seizure control; and 2 or more AED to effect control. Independent risk factors for relapse were: 6 years of age or higher at onset, and 5 years or more from start of treatment to seizure control. These risk factors are of value in attempting AED withdrawal.

\section{ATTENTION DEFICIT DISORDERS}

\section{EFFECTS OF METHYLPHENIDATE ON ATTENTION IN ADHD}

In a randomized, double-blind, placebo-controlled study of 60 children aged 8-12 years with ADHD treated with two doses of methylphenidate (MPH), 0.25 and $0.5 \mathrm{mg} / \mathrm{kg}$, and placebo, a linear improvement was obtained at both doses in alertness and focused and sustained attention. No significant improvement occurred for divided attention. Intensitydimension functions are best influenced by higher doses, executive functions by moderate doses, and selective-dimension functions by variable doses. Responders defined by improved behavior did not differ from nonresponders. (Konrad K, Gunther T, Hanisch C, HerpertzDahlmann B. J Am Acad Child Adolesc Psychiatry Feb 2004;43:191-298). 